PROCEEDINGS OF THE

AMERICAN MATHEMATICAL SOCIETY

Volume 138, Number 9, September 2010, Pages 3057-3061

S 0002-9939(2010)10279-4

Article electronically published on May 14, 2010

\title{
LAMPLIGHTER GRAPHS DO NOT ADMIT HARMONIC FUNCTIONS OF FINITE ENERGY
}

\author{
AGELOS GEORGAKOPOULOS
}

(Communicated by Jim Haglund)

\begin{abstract}
We prove that a lamplighter graph of a locally finite graph over a finite graph does not admit a non-constant harmonic function of finite Dirichlet energy.
\end{abstract}

\section{INTRODUCTION}

The wreath product $G$ \{ $H$ of two groups $G, H$ is a well-known concept. Cayley graphs of $G$ ? $H$ can be obtained in an intuitive way by starting with a Cayley graph of $G$ and associating with each of its vertices a lamp whose possible states are indexed by the elements of $H$; see below. Graphs obtained this way are called lamplighter graphs. A well-known special case are the Diestel-Leader [7] graphs $D L(n, n)$.

Kaimanovich and Vershik [11, Sections 6.1, 6.2] proved that lamplighter graphs of infinite grids $\mathbb{Z}^{d}, d \geq 3$ admit non-constant, bounded, harmonic functions. Their construction had an intuitive probabilistic interpretation related to random walks on these graphs, which triggered a lot of further research on lamplighter graphs. For example, spectral properties of such groups are studied in [5, 10, 13, and other properties related to random walks are studied in 8, 9, 17. Harmonic functions on lamplighter graphs and the related Poisson boundary are further studied e.g. in [3, 12, 18. Finally, Lyons, Pemantle and Peres 14 proved that the lamplighter graph of $\mathbb{Z}$ over $\mathbb{Z}_{2}$ has the surprising property that random walk with a drift towards a fixed vertex can move outwards faster than simple random walk.

It is known that the existence of a non-constant harmonic function of finite Dirichlet energy implies the existence of a non-constant bounded harmonic function [19. Theorem 3.73]. Given the aforementioned impact that bounded harmonic functions on lamplighter graphs have had, we ask whether these graphs have nonconstant harmonic functions of finite Dirichlet energy. For lamplighter graphs on a grid it is known that no such harmonic functions can exist, since such graphs are amenable and thus admit no non-constant harmonic functions of finite Dirichlet energy 16. A. Karlsson (oral communication) asked whether this is also the case for graphs of the form $T \nmid \mathbb{Z}_{2}$, where $T$ is any regular tree. In this paper we give an affirmative answer to this question. In fact, the actual result is much more general:

Received by the editors August 12, 2009.

2010 Mathematics Subject Classification. Primary 05C25.

The author was supported by FWF grant P-19115-N18.

(C)2010 Agelos Georgakopoulos 
Theorem 1.1. Let $G$ be a connected locally finite graph and let $H$ be a connected finite graph with at least one edge. Then $G$ ? $H$ does not admit any non-constant harmonic function of finite Dirichlet energy.

Indeed, we do not need to assume that any of the involved graphs is a Cayley graph. Lamplighter graphs on general graphs can be defined as in the usual case when all graphs are Cayley graphs; see the next section.

It is easy to prove, and well-known, that the non-existence of non-constant harmonic functions in a graph is equivalent to the uniqueness of electrical currents. Thus, in a lamplighter graph $G \imath H$ as in Theorem [1.1 electrical currents of finite energy are unique.

Classes of graphs that do admit non-constant harmonic functions of finite Dirichlet energy are known; see [1, 2, 4.

As an intermediate step to the proof of Theorem 1.1 we prove a result (Lemma 3.1 below) that strengthens a theorem of Markvorsen, McGuinness and Thomassen [15. and might be applicable in order to prove that other classes of graphs do not admit non-constant Dirichlet-finite harmonic functions.

\section{Definitions}

We will be using the terminology of Diestel [6]. For a finite path $P$ we let $|P|$ denote the number of edges in $P$. For a graph $G$ and a set $U \subseteq V(G)$, we let $G[U]$ denote the subgraph of $G$ induced by the vertices in $U$. If $G$ is finite, then its diameter $\operatorname{diam}(G)$ is the maximum distance, in the usual graph metric, of two vertices of $G$.

Let $G, H$ be connected graphs, and suppose that every vertex of $G$ has a distinct lamp associated with it, the set of possible states of each lamp being the set of vertices $V(H)$ of $H$. At the beginning, all lamps have the same state $s_{0} \in V(H)$, and a "lamplighter" is standing at some vertex of $G$. In each unit of time the lamplighter is allowed to choose one of two possible moves: either walk to a vertex of $G$ adjacent to the vertex $x \in V(G)$ he is currently at or switch the current state $s \in V(H)$ of $x$ into one of the states $s^{\prime} \in V(H)$ adjacent with $s$. The lamplighter graph $G$ ? $H$ is, then, a graph whose vertices correspond to the possible configurations of this game and whose edges correspond to the possible moves of the lamplighter. More formally, the vertex set of $G \imath H$ is the set of pairs $(C, x)$ where $C: V(G) \rightarrow V(H)$ is an assignment of states such that $C(v) \neq s_{0}$ holds for only finitely many vertices $v \in V(G)$, and $x$ is a vertex of $G$ (the current position of the lamplighter). Two vertices $(C, x)$ and $\left(C^{\prime}, x^{\prime}\right)$ of $G \imath H$ are joined by an edge if (precisely) one of the following conditions holds:

- $C=C^{\prime}$ and $x x^{\prime} \in E(G)$, or

- $x=x^{\prime}$, all vertices except $x$ are mapped to the same state by $C$ and $C^{\prime}$, and $C(x) C^{\prime}(x) \in E(H)$.

This definition of $G \imath H$ coincides with that of Erschler [9].

The blow-up of a vertex $v \in V(G)$ in $L=G \imath H$ is the set of vertices of $L$ of the form $(C, v)$. Similarly, the blow-up of a subgraph $T$ of $G$ is the subgraph of $L$ spanned by the blow-ups of the vertices of $T$. Given a vertex $x \in V(L)$ we let $[x]$ denote the vertex of $G$, the blow-up of which contains $x$.

An edge of $L$ is a switching edge if it corresponds to a move of the lamplighter that switches a lamp, more formally, if it is of the form $(C, v)\left(C^{\prime}, v\right)$. For a switching 
edge $e \in E(L)$, we let $[e]$ denote the corresponding edge of $H$. A ray is a 1-way infinite path; a 2-way infinite path is called a double ray. A tail of a ray $R$ is an infinite (co-final) subpath of $R$.

A function $\phi: V(G) \rightarrow \mathbb{R}$ is harmonic if for every $x \in V(G)$ we have $\phi(x)=$ $\frac{1}{d(x)} \sum_{x y \in E(G)} \phi(y)$, where $d(x)$ is the number of edges incident with $x$. Given such a function $\phi$, and an edge $e=u v$, we let $w_{\phi}(e):=(\phi(u)-\phi(v))^{2}$ denote the energy dissipated by $e$. The (Dirichlet) energy of $\phi$ is defined by $W(\phi):=\sum_{e \in E(G)} w_{\phi}(e)$.

\section{Proof of Theorem 1.1}

We start with a lemma that might be applicable in order to prove that other classes of graphs do not admit non-constant Dirichlet-finite harmonic functions. This strengthens a result of [15, Theorem 7.1].

Lemma 3.1. Let $G$ be a connected locally finite graph such that for every two disjoint rays $S, Q$ in $G$ there is a constant $c$ and a sequence $\left(P_{i}\right)_{i \in \mathbb{N}}$ of pairwise edge-disjoint $S-Q$ paths such that $\left|P_{i}\right| \leq c i$. Then $G$ does not admit a non-constant harmonic function of finite energy.

Proof. Let $G$ be a locally finite graph that admits a non-constant harmonic function $\phi$ of finite energy; it suffices to find two rays $S, Q$ in $G$ that do not satisfy the condition in the assertion.

Since $\phi$ is non-constant, we can find an edge $x_{0} x_{1}$ satisfying $\phi\left(x_{1}\right)>\phi\left(x_{0}\right)$. By the definition of a harmonic function, it is easy to see that $x_{0} x_{1}$ must lie in a double ray $D=\ldots x_{-1} x_{0} x_{1} \ldots$ such that $\phi\left(x_{i}\right) \geq \phi\left(x_{i-1}\right)$ for every $i \in \mathbb{Z}$; indeed, every vertex $x \in V(G)$ must have a neighbour $y$ such that $\phi(y) \geq \phi(x)$.

Define the subrays $S=x_{0} x_{1} x_{2} \ldots$ and $Q=x_{0} x_{-1} x_{-2} \ldots$ of $D$. Now suppose there is a sequence $\left(P_{i}\right)_{i \in \mathbb{N}}$ of pairwise edge-disjoint $S-Q$ paths such that $\left|P_{i}\right| \leq c i$ for some constant $c$.

Note that by the choice of $D$ there is a bound $u>0$ such that $u_{i}:=\mid \phi\left(s_{i}\right)-$ $\phi\left(q_{i}\right) \mid \geq u$ for every $i$, where $s_{i} \in V(S)$ and $q_{i} \in V(Q)$ are the endvertices of $P_{i}$.

For every edge $e=x y$ let $f(e):=|\phi(y)-\phi(x)|$. Let $X_{i}$ be the set of edges $e$ in $P_{i}$ such that $f(e) \geq 0.9 \frac{u}{c i}$, and let $Y_{i}$ be the set of all other edges in $P_{i}$. As $\left|P_{i}\right| \leq c i$ by assumption, the edges in $Y_{i}$ contribute less than $0.9 u$ to $u_{i}$; thus $\sum_{e \in X_{j}} f(e)>0.1 u$ must hold. But since $f(e) \geq 0.9 \frac{u}{c i}$ for every $e \in X_{j}$, we have $\sum_{e \in X_{j}} w_{\phi}(e)>0.1 \times 0.9 \frac{u^{2}}{c i}$. As the sets $X_{j}$ are pairwise edge-disjoint, and as the series $\sum_{i} 1 / i$ is not convergent, this contradicts the fact that $\sum_{e \in E(G)} w_{\phi}(e)$ is finite.

We now apply Lemma 3.1 to prove our main result.

Proof of Theorem 1.1. We will show that $L:=G \geq H$ satisfies the condition of Lemma 3.1, from which then the assertion follows. So let $S, Q$ be any two disjoint rays of $L$.

Since $L$ is connected we can find a double ray $D$ in $L$ that contains a tail $S^{\prime}$ of $S$ and a tail $Q^{\prime}$ of $Q$. Let $s_{0}$ (respectively, $q_{0}$ ) be the first vertex of $S^{\prime}$ (resp. $Q^{\prime}$ ). Let $V_{0}$ be the set of vertices of $G$, the blow-up of which meets the path $s_{0} D q_{0}$. Note that $V_{0}$ induces a connected subgraph of $G$, because the lamplighter only moves along the edges of $G$. Thus we can choose a spanning tree $T_{0}$ of $G\left[V_{0}\right]$.

For $i=1,2, \ldots$ we construct an $S^{\prime}-Q^{\prime}$ path $P_{i}$ as follows. Let $s_{i}$ be the first vertex of $S^{\prime}$ not in the blow-up of $V_{i-1}$, and let $q_{i}$ be the first vertex of $Q^{\prime}$ not in 
the blow-up of $V_{i-1}$. Let $V_{i}:=V_{i-1} \cup\left\{s_{i}, q_{i}\right\}$, and extend $T_{i-1}$ into a spanning tree $T_{i}$ of $G\left[V_{i}\right]$ by adding two edges incident with $s_{i}$ and $q_{i}$, respectively; such edges do exist: their blow-up contains the edges of $S^{\prime}, Q^{\prime}$ leading into $s_{i}, q_{i}$ respectively.

We now construct an $s_{i}-q_{i}$ path $P_{i}$. Pick a switching edge $e=s_{i} s_{i}^{\prime}$ incident with $s_{i}$. Then let $X_{i}$ be the unique path in $L$ from $s_{i}^{\prime}$ to a vertex $q_{i}^{+}$with $\left[q_{i}^{+}\right]=\left[q_{i}\right]$ such that $X_{i}$ is contained in the blow-up of $T_{i}$. Pick a switching edge $f=q_{i}^{+} q_{i}^{-}$incident with $q_{i}^{+}$. Then follow the unique path $Y_{i}$ in $L$ from $q_{i}^{-}$to a vertex $s_{i}^{+}$with $\left[s_{i}^{+}\right]=\left[s_{i}\right]$ such that $Y_{i}$ is contained in the blow-up of $T_{i}$. Let $e^{\prime}=s_{i}^{+} s_{i}^{-}$be the switching edge incident with $s_{i}^{+}$such that $\left[e^{\prime}\right]=[e]$. Finally, let $Z_{i}$ be a path from $s_{i}^{-}$to the unique vertex $q_{i}^{\prime}$ with $\left[q_{i} q_{i}^{\prime}\right]=[f]$, such that the interior of $Z_{i}$ is contained in the blow-up of $V_{i-1}$ and $Z_{i}$ has minimum length under all paths with these properties. Such a path exists because every lamp at a vertex in $G-V_{i-1}$ has the same state in $s_{i}^{-}$and $q_{i}^{\prime}$; indeed, the lamps in $G-V_{i}$ were never switched in the above construction, the lamp at $\left[s_{i}\right]$ was switched twice on the way from $s_{i}$ to $s_{i}^{-}$using the same switching edge [e], which means that its state in both endpoints of $Z_{i}$ coincides with that in $s_{i}$ and $q_{i}$, and finally the lamp at $\left[q_{i}^{\prime}\right]$ has the same state in both endpoints of $Z_{i}$, namely the state $[f]$ leads to. Now set $P_{i}:=s_{i} s_{i}^{\prime} X_{i} q_{i}^{+} q_{i}^{-} Y_{i} s_{i}^{+} s_{i}^{-} Z_{i} q_{i}^{\prime} q_{i}$.

It is not hard to check that the paths $P_{i}$ are pairwise disjoint. Indeed, let $i<j \in \mathbb{N}$. Then, by the choice of the vertices $s_{j}, q_{j}$ and the construction of $P_{j}$, it follows that for every inner vertex $x$ of $P_{j}$, the configuration of $x$ differs from the configuration of any vertex in $P_{i}$ in at least one of the two lamps at $\left[s_{j}\right]$ and $\left[q_{j}\right]$.

It remains to show that there is a constant $c$ such that $\left|P_{i}\right| \leq c i$ for every $i$. To prove this, note that $\left|P_{i}\right|=\left|X_{i}\right|+\left|Y_{i}\right|+\left|Z_{i}\right|+4$; we will show that the latter three subpaths grow at most linearly with $i$, which then implies that this is also true for $P_{i}$.

Firstly, note that $\operatorname{diam}\left(T_{i}\right)-\operatorname{diam}\left(T_{i-1}\right) \leq 2$ since $V\left(T_{i}\right):=V\left(T_{i-1}\right) \cup\left\{s_{i}, q_{i}\right\}$. By the choice of $X_{i}$ we have $\left|X_{i}\right| \leq \operatorname{diam}\left(T_{i}\right)$, from which it follows that there is a constant $c_{1}$ such that $\left|X_{i}\right| \leq c_{1} i$. By the same argument, we have $\left|Y_{i}\right| \leq c_{1} i$.

It remains to bound the length of $Z_{i}$. For this, note that if $T$ is a finite tree and $v, w \in V(T)$, then there is a $v-w$ walk $W$ in $T$ containing all edges of $T$ and satisfying $|W| \leq 3|E(T)|$; indeed, starting at $v$, one can first walk around the "perimeter" of $T$ traversing every edge precisely once in each direction $(2|E(T)|$ edges), and then move "straight" from $v$ to $w$ (at most $|E(T)|$ edges). Thus, in order to choose $Z_{i}$, we could put a lamplighter at the vertex and configuration indicated by $s_{i}^{-}$and let him move in $T_{i} \subset G$ along such a walk $W$ from $\left[s_{i}^{-}\right]$to $\left[q_{i}^{\prime}\right]$, and every time he visits a new vertex $x$ let him change the state of $x$ to the state indicated by $q_{i}^{\prime}$. This bounds the length of $Z_{i}$ from above by $3\left|E\left(T_{i}\right)\right| \operatorname{diam}(H)$, and since $\left|E\left(T_{i}\right)\right|-\left|E\left(T_{i-1}\right)\right|=2$ and $H$ is fixed, we can find a constant $c_{2}$ such that $\left|Z_{i}\right| \leq c_{2} i$ for every $i$. This completes the proof that $\left|P_{i}\right|$ grows at most linearly with $i$.

Thus we can now apply Lemma 3.1 to prove that $G \imath H$ does not admit a nonconstant harmonic function of finite energy.

Problem 3.1. Does the assertion of Theorem 1.1 still hold if $H$ is an infinite locally finite graph?

Lemma 3.1 might be applicable in order to prove that other classes of graphs also do not admit non-constant Dirichlet-finite harmonic functions. For example, it yields an easy proof of the (well-known) fact that infinite grids have this property. 


\section{ACKNOWLEDGEMENTS}

I am grateful to Jörg Lehnert for pointing out some errors in an earlier version of this paper and to Wolfgang Woess for suggesting the problem and helpful discussions.

\section{REFERENCES}

1. M. E. B. Bekka and A. Valette. Group cohomology, harmonic functions and the first $L^{2}$-Betti number. Potential Anal., 6(4):313-326, 1997. MR1452785 (98e:20056)

2. I. Benjamini and O. Schramm. Harmonic functions on planar and almost planar graphs and manifolds, via circle packings. Invent. Math., 126:565-587, 1996. MR 1419007 (97k:31009)

3. S. Brofferio and W. Woess. Positive harmonic functions for semi-isotropic random walks on trees, lamplighter groups, and DL-graphs. Potential Anal., 24(3):245-265, 2006. MR 2217953 (2007b:31010)

4. D. I. Cartwright and W. Woess. Infinite graphs with nonconstant Dirichlet finite harmonic functions. SIAM J. Discrete Math., 5(3):380-385, 1992. MR1172746 (94a:31005)

5. W. Dicks and T. Schick. The spectral measure of certain elements of the complex group ring of a wreath product. Geom. Dedicata, 93:121-137, 2002. MR.1934693 (2003i:20005)

6. R. Diestel. Graph Theory (3rd edition). Springer-Verlag, 2005. Electronic edition available at: http://www.math.uni-hamburg.de/home/diestel/books/graph.theory. MR2159259 (2006e:05001)

7. R. Diestel and I. Leader. A conjecture concerning a limit of non-Cayley graphs. J. Algebraic Combinatorics, 14:17-25, 2001. MR:1856226 (2002h:05082)

8. A. Erschler. On drift and entropy growth for random walks on groups. Ann. Probab., 31(3):1193-1204, 2003. MR1988468 (2004c:60018)

9. A. Erschler. Generalized wreath products. Int. Math. Res. Not., 2006:1-14, 2006. MR 2276348 (2008c:05080)

10. R.I. Grigorchuk and A. Zuk. The lamplighter group as a group generated by a 2-state automaton, and its spectrum. Geom. Dedicata, 87(1-3):209-244, 2001. MR1866850 (2002j:60009)

11. V.A. Kaimanovich and A.M. Vershik. Random walks on discrete groups: Boundary and entropy. Ann. Probab., 11:457-490, 1983. MR704539 (85d:60024)

12. A. Karlsson and W. Woess. The Poisson boundary of lamplighter random walks on trees. Geom. Dedicata, 124:95-107, 2007. MR2318539(2009b:60246)

13. F. Lehner, M. Neuhauser, and W. Woess. On the spectrum of lamplighter groups and percolation clusters. Mathematische Annalen, 342:69-89, 2008. MR2415315 (2009d:60329)

14. R. Lyons, R. Pemantle, and Y. Peres. Random walks on the lamplighter group. The Annals of Probability, 24(4):1993-2006, 1996. MR1415237 (97j:60014)

15. S. Markvorsen, S. McGuinness, and C. Thomassen. Transient random walks on graphs and metric spaces with applications to hyperbolic surfaces. Proc. London Math. Soc., 64:1-20, 1992. MR 1132852 (93e:60142)

16. G. Medolla and P.M. Soardi. Extension of Foster's averaging formula to infinite networks with moderate growth. Math. Z., 219(2):171-185, 1995. MR1337213 (96g:94031)

17. C. Pittet and L. Saloff-Coste. On random walks on wreath products. Ann. Probab., 30(2):948977, 2002. MR1905862 (2003d:60013)

18. Ecaterina Sava. A note on the Poisson boundary of lamplighter random walks. Monatshefte für Mathematik. 159 (2010), 329-344.

19. P.M. Soardi. Potential theory on infinite networks., volume 1590 of Lecture Notes in Math., Springer-Verlag, 1994. MR,1324344(96i:31005)

Technische Universität Graz, Steyrergasse 30, 8010, Graz, Austria 